\title{
La biofortificación con selenio mejora la calidad nutracéutica y la capacidad antioxidante de la lechuga (Lactuca sativa L.)
}

\section{Selenium biofortification improves nutraceutical quality and antioxidant capacity of lettuce (Lactuca sativa L.)}

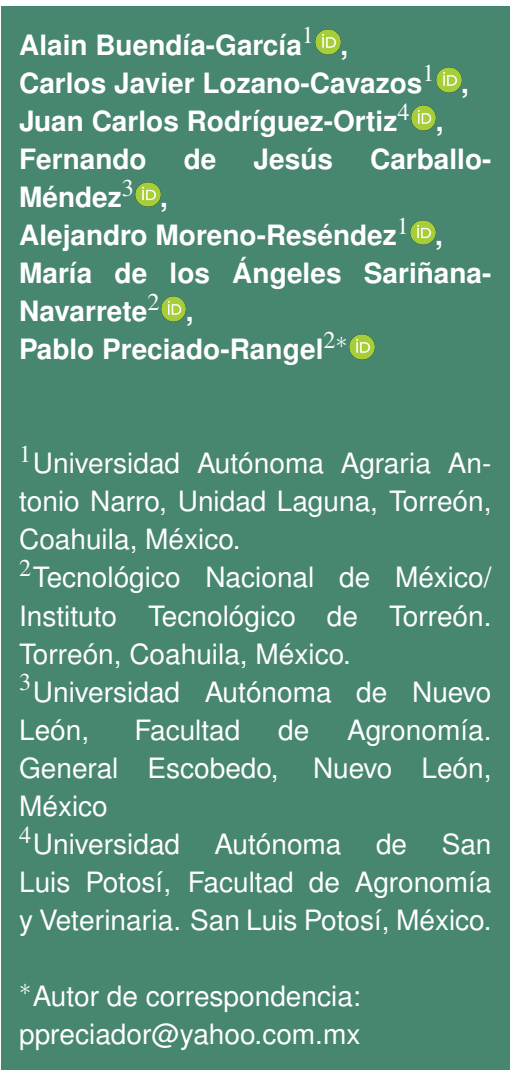

Nota científica

Recibida: 11 de Agosto 2021 Aceptada: 23 de septiembre 2021

Como citar: Buendía-García A Lozano-Cavazos CJ, Rodríguez-Ortiz JC, Carballo-Méndez FJ, MorenoReséndez A, Sariñana-Navarrete MA, Preciado-Rangel P (2021) La biofortificación con selenio mejora la calidad nutracéutica y la capacidad antioxidante de la lechuga (Lactuca sativa L.). Ecosistemas y Recursos Agropecuarios 8(3): e3104. DOI: 10.19136/era.a8n3.3104
RESUMEN. EI Selenio (Se), es un micronutriente esencial para los humanos y su deficiencia afecta a un $15 \%$ de la población mundial. La biofortificación agronómica mejora la biosíntesis de compuestos bioactivos y propicia su bioacumulación. En el presente trabajo se evaluó el efecto de la aplicación foliar de Se sobre el rendimiento, biosíntesis de compuestos bioactivos y acumulación en lechuga (Lactuca sativa L.) cv "Parris Island cos". Cinco tratamientos fueron utilizados: $0,10,20,40$ y $60 \mu \mathrm{M} \mathrm{L}^{-1}$. La biofortificación con Se modificó positivamente la biosíntesis de compuestos fitoquímicos y su concentración en hojas de lechuga, sin afectar el rendimiento. La aspersión foliar de Se es una alternativa para incrementar la biosíntesis de compuestos fitoquímicos e incrementar la concentración de este elemento en lechuga con la posibilidad de mejorar la salud pública con su consumo.

Palabras clave: Selenato, rendimiento, compuestos fitoquímicos.

ABSTRACT. Selenium (Se) is an essential micronutrient for humans and its deficiency affects $15 \%$ of the world's population. Agronomic biofortification improves the biosynthesis of bioactive compounds and promotes their bioaccumulation. In the present work, the effect of foliar application of Se on yield, biosynthesis of bioactive compounds and accumulation in lettuce (Lactuca sativa L.) cv "Parris Island cos". was evaluated. Five treatments were used: $0,10,20,40$ and $60 \mu \mathrm{M} \mathrm{L}^{-1}$. Biofortification with the biosynthesis of phytochemical compounds and their concentration in lettuce leaves was positively modified, without affecting the yield. Se foliar spraying is an alternative to increase the biosynthesis of phytochemical compounds and increase the concentration of this element in lettuce with the possibility of improving public health with its consumption.

Key words: Selenate, yield, phytochemical compounds. 


\section{INTRODUCCIÓN}

El Selenio (Se) es un oligoelemento esencial en la nutrición humana, participando en varias funciones dentro del organismo que mantienen el crecimiento y adecuado funcionamiento de las células (Schiavon et al. 2020). Este elemento es un cofactor de la enzima glutatión peroxidasa, la cual es un catalizador en la reducción de peróxidos que pueden dañar las células y tejidos, además puede actuar como antioxidante (Puccinelli et al. 2017). Las formas de Se que están biodisponibles para el ser humano son los selenoaminoácidos (Selenometionina SeMet y Selenocisteína SeCys), estos aminoácidos forman parte constituyente de las selenoproteínas, las cuales tienen un papel importante en sistemas antioxidantes, equilibrio hormonal, inmunidad, fertilidad masculina, resistencia a infecciones virales y prevención de cáncer (Rayman 2020). Las selenoproteínas necesitan varios cofactores para su síntesis y dependen principalmente de la ingesta del Se contenido en la carne o pescado (Willers et al. 2015), debido a que los alimentos de origen vegetal presentan un bajo contenido de este oligoelemento (Kleine-Kalmer et al. 2021), como un reflejo de la concentración de este elemento en el suelo (White 2016), lo anterior se manifiesta como una deficiencia de Se que afecta al 15\% de la población mundial (Wu et al. 2015, GarcíaMárquez et al. 2020). Una estrategia para aumentar el contenido de Se en los alimentos de origen vegetal es mediante la biofortificación que consiste en potenciar la bioactividad y el contenido de Se en las partes comestibles de las plantas (Gaucín-Delgado et al. 2020). La fertilización foliar y edáfica, son los principales métodos para la suplementación de Se a los cultivos; sin embargo, debido a la baja eficiencia de la aplicación al suelo, la aspersión foliar con Se, es considerado como el método más efectivo para el enriquecimiento de los cultivos con micronutrientes (Deng et al. 2019). Por otro lado, la lechuga (Lactuca sativa L.) es una de las hortalizas de hoja más consumidas en el mundo, principalmente en fresco (Medina-Lozano et al. 2021), es rica en fibra, ácidos grasos, aminoácidos, proteínas y minerales (Kim et al. 2016). Además, posee compuestos fitoquímicos (Lee et al. 2021), que aportan propiedades antioxidantes (Zapata-Vahos et al. 2020). La aplicación de micronutrientes a través de la biofortificación de cultivos es una herramienta útil no solo para incrementar la cantidad de minerales sino, además, se mejora considerablemente la producción de compuestos bioactivos. Por tanto, el objetivo de este trabajo fue determinar el efecto de la biofortificación foliar con Selenio sobre el rendimiento, la calidad nutracéutica y la capacidad antioxidante en el cultivo de lechuga.

\section{MATERIALES Y MÉTODOS}

\section{Material vegetal y tratamientos}

El estudio se realizó en un invernadero circular ubicado en el Instituto Tecnológico de Torreón, Coahuila, México, localizado geográficamente a $24^{\circ}$ $30^{\prime}$ de latitud norte, $102^{\circ} 00^{\prime}$ de longitud oeste y altitud de 1120 metros sobre el nivel del mar. Semilla de lechuga (Lactuca sativa L.) "Parris Island cos" de Heirloom Seeds ${ }^{\circledR}$ fueron germinadas en placas de foamy agrícola. Treinta días después de la siembra las plántulas se trasplantaron a un sistema hidropónico NFT y la nutrición del cultivo se realizó utilizando la solución nutritiva Steiner (Steiner 1961). Los tratamientos evaluados consistieron en la aplicación foliar de selenato de sodio $\left(\mathrm{Na}_{2} \mathrm{SeO}_{4}\right.$. $95 \%$ de pureza, Sigma-Aldrich $\left.{ }^{\circledR}\right)$ : 0, 10, 20, 40 y $60 \mu$ $\mathrm{M} \mathrm{L}^{-1}$, utilizando agua destilada como diluyente $y$ un surfactante comercial no tóxico (INEX-A ${ }^{\circledR}$, $2 \mathrm{~mL}$ $\mathrm{L}^{-1}$ ). Las aplicaciones foliares se realizaron con aspersores manuales durante las primeras horas de la mañana, en total se realizaron tres aspersiones cada 15 días después del trasplante.

\section{Muestreo}

Las lechugas se cosecharon 60 días después del trasplante, se midieron y se pesaron para determinar las variables respuesta del rendimiento y bioquímicas, así como el contenido de Se en hojas de lechuga, obteniendo seis muestras por cada tratamiento. 


\section{Rendimiento}

Para determinar el peso fresco (rendimiento del cultivo), las cabezas de lechuga se cosecharon y se pesaron en una balanza analítica (Ohaus Corporation, Pine Brook, Nueva Jersey, EE. UU).

\section{Análisis proximal de lechuga}

El contenido de proteína se determinó con el método Dumas (Calvo et al. 2008), el contenido de fibra cruda se determinó de acuerdo a la NOM-F90-S-1978, el contenido de grasa se determinó con el método Goldfish de acuerdo a la NMX-F-427-1982.

\section{Determinación de selenio}

Las muestras secas se trituraron en un mortero de porcelana y se digirieron con ácido nítrico y perclórico (3:1), utilizando una placa calefactora a 100 ${ }^{\circ} \mathrm{C}$. La solución se filtró y se hirvió para obtener 100 $\mathrm{mL}$ de solución de trabajo con agua desionizada. La concentración de selenio en hojas de lechuga se determinó mediante espectrofotometría de absorción atómica (Helrich 1990), los resultados se expresaron en $\mu \mathrm{g} \mathrm{kg}^{-1}$ de peso seco de hojas de lechuga.

\section{Compuestos bioactivos}

Para los extractos etanólicos, se molieron 100 $\mathrm{g}$ de pulpa de lechuga fresca por tratamiento y se utilizaron para determinar la calidad nutracéutica de la lechuga. Se colocó $1 \mathrm{~g}$ de muestra en un tubo Falcon de $15 \mathrm{~mL}$ y se agregaron $10 \mathrm{~mL}$ de etanol grado reactivo. Después de 1 min de agitación en Vortex, se dejaron reposar durante $24 \mathrm{~h}$. Los extractos etanólicos se decantaron posteriormente a $3500 \mathrm{x} g$ y el sobrenadante se transfirió a un tubo Falcon y se almacenó a $-20{ }^{\circ} \mathrm{C}$ hasta su uso.

El contenido fenólico total se determinó mediante una modificación del método de FolinCiocalteau (García-Nava, 2009). Se tomaron $150 \mu \mathrm{L}$ de extracto etanólico, se diluyeron en $3 \mathrm{~mL}$ de agua (milli-Q), se agregaron $250 \mu \mathrm{L}$ de reactivo de FolinCiocalteau $(1 \mathrm{~N})$, se agitó y se dejó reaccionar durante $3 \mathrm{~min}$. Posteriormente se añadieron $750 \mu \mathrm{L}$ de $\mathrm{Na}_{2} \mathrm{CO}_{3}(20 \%)$ y $950 \mu \mathrm{L}$ de agua (milli-Q). Se dejó reposar la solución durante $2 \mathrm{~h}$ y se cuantificaron las muestras en un espectrofotómetro UV-Vis a $760 \mathrm{~nm}$.
El patrón se preparó con ácido gálico. Los resultados se expresaron en mg GAE $100 \mathrm{~g}^{-1}$ de peso fresco.

Los flavonoides totales se determinaron por colorimetría (García-Nava, 2009). Se tomaron 200 $\mu \mathrm{L}$ de extracto etanólico, se mezclaron con $1,25 \mathrm{~mL}$ de agua (milli-Q) y $75 \mu \mathrm{L}$ de $\mathrm{NaNO}_{2}$ (5\%). Después de $5 \mathrm{~min}$ de reposo, se agregaron $150 \mu \mathrm{L}$ de $\mathrm{AlCl} 3$. Posteriormente, se añadieron $500 \mu \mathrm{L}$ de $\mathrm{NaOH}(1$ M) y $275 \mu \mathrm{L}$ de agua (milli-Q). Se agitó vigorosamente y las muestras se cuantificaron en un espectrofotómetro UV-Vis a $510 \mathrm{~nm}$. El estándar se preparó con quercetina disuelta en etanol absoluto (y $\left.=0.0122 x-0.0067 ; r^{2}=0.965\right)$. Los resultados se expresaron en mg QE $100 \mathrm{~g}^{-1}$ de peso fresco.

La capacidad antioxidante total se midió mediante el método DPPH + in vitro (Brand-Williams et al. 1995). Se preparó una solución de $\mathrm{DPPH}^{+}$ (Aldrich, St. Louis, Missouri, EE. UU.) en etanol, a una concentración de $0,025 \mathrm{mg} \mathrm{mL}^{-1}$. Se mezclaron $700 \mu \mathrm{L}$ de extracto etanólico con $1.300 \mu \mathrm{L}$ de solución $\mathrm{DPPH}^{+}$, después de 30 min las muestras se cuantificaron en un espectrofotómetro UV-Vis a $517 \mathrm{~nm}$. Los resultados se expresaron en $\mu \mathrm{M}$ equivalente en Trolox $100 \mathrm{~g}^{-1}$ de peso fresco.

\section{Análisis estadístico}

El diseño experimental fue completamente al azar con seis repeticiones por tratamiento, considerándose una planta como unidad experimental. Las variables respuesta fueron analizadas mediante un análisis de varianza para determinar las diferencias entre los tratamientos y donde se detectó diferencia significativa, se utilizó la prueba Tukey ( $P$ $\leq 0.05)$.

\section{RESULTADOS Y DISCUSIÓN}

\section{Rendimiento}

La aspersión foliar de Se en las dosis utilizadas no afectó el peso fresco de la lechuga (Tabla 1). Debido a que él Se no es considerado esencial para el metabolismo de las plantas, no se esperaría que la suplementación con Se cause cambios en el crecimiento y rendimiento de los cultivos (Hossain et 
Tabla 1. Efecto de la aplicación foliar de $\mathrm{Na}_{2} \mathrm{SeO}_{4}$ sobre el peso fresco y composición proximal de lechuga.

\begin{tabular}{lcccc}
\hline $\begin{array}{l}\mathrm{Na}_{2} \mathrm{SeO}_{4} \\
\mu \mathrm{M} \mathrm{L}^{-1}\end{array}$ & $\begin{array}{c}\text { Peso fresco } \\
\mathrm{g}\end{array}$ & \begin{tabular}{c} 
Grasa cruda \\
\cline { 2 - 5 }
\end{tabular} & $\begin{array}{c}\text { Proteína cruda } \\
\%\end{array}$ & Fibra cruda \\
\hline Control & 568.2 & $3,14^{c}$ & $14.55^{e}$ & $16.63^{d}$ \\
10 & 769.7 & $3,44^{b c}$ & $17.44^{b}$ & $16.04^{e}$ \\
20 & 771.7 & $5.15^{a}$ & $18.28^{a}$ & $17.61^{a}$ \\
40 & 667.7 & $3.69^{b}$ & $16.36^{c}$ & $17.05^{c}$ \\
60 & 656.9 & $4.8^{a}$ & $15.05^{d}$ & $17.21^{b}$ \\
\hline${ }^{*}$ Letras diferentes indican una diferencia significativa según la prueba de Tukey \\
(P $\leq 0.05)$
\end{tabular}

al. 2021). Sin embargo, existen reportes contradictorios al respecto, ya que él Se en dosis bajas afecta positivamente el rendimiento (Zhu et al. 2017) y altas dosis causan toxicidad en las plantas debido a la alta producción de especies reactivas de oxígeno, que inhiben el crecimiento y el rendimiento (Lapaz et al. 2019). Los resultados son consistentes con los trabajos reportados por Wang et al. (2020) en Triticum aestivum $L$ y Rahim et al. (2020) en Solanum lycopersicon L, quienes tampoco encontraron cambios en el rendimiento. En general la respuesta de la planta al Se depende de la concentración utilizada (Gaucín-Delgado et al. 2020); la sensibilidad de la especie (El-Ramady et al. 2020), la especie química utilizada y el método de aplicación, por lo que no existe una dosis única para todos los cultivos (Oliveira et al. 2018).

\section{Composición proximal}

Los resultados obtenidos muestran que la aspersión foliar con Se causó cambios significativos ( $P$ $\leq 0.05$ ), en el contenido de grasa, proteína y fibra cruda (Tabla 1). Se aprecia que los mayores valores de estos parámetros fueron obtenidos con la aspersión foliar de $20 \mu \mathrm{M} \mathrm{L}^{-1}$. Los incrementos de este tratamiento con relación al testigo fueron de 20.4, 5.6 y $39 \%$ para el contenido de grasa, proteínas y fibra cruda, respectivamente. La utilización de Se en los programas de biofortificación es una buena estrategia agronómica; debido a que además de incrementar el contenido de oligoelementos esenciales para la nutrición humana, mejora sustancialmente la calidad proximal de los vegetales ya que la adecuada proporción de fibra, proteína y grasa en algunos vegetales de hoja verde sugieren su potencial como agentes anabólicos y como fuentes alternativas de energía (Moussa et al. 2020). Las proteínas consumidas en la dieta humana proveen un aminoácido que el cuerpo no es capaz de producir (Khan et al. 2019). La grasa cruda determina los grasos lípidos libres y puede modificar el sabor de los alimentos (Emelike et al. 2020). Adicionalmente, un alto contenido de fibra en la dieta diaria reduce los niveles de colesterol en el cuerpo, resultando en la baja incidencia de enfermedades cardiovasculares (Chatepa y Masamba 2020). El incremento de las proteínas probablemente sea debido a una mayor síntesis de las selenoproteínas (selenometionina (SeMet) y la selenocisteína (SeCys), las cuales dependen principalmente del Se (Gupta y Gupta 2017).

\section{Calidad nutracéutica}

La calidad nutracéutica de lechuga determinada por los compuestos fenólicos, flavonoides totales y la capacidad antioxidante, fue afectada significativamente $(P \leq 0.05)$ por la aspersión foliar de Se. Los mayores valores se lograron con la aspersión foliar de $60 \mu \mathrm{M} \mathrm{L}^{-1}$, superando $21.68,105.11$ y $8.6 \%$ en la cuantificación de fenoles totales, flavonoides y actividad antioxidante en las plantas del tratamiento testigo (Figura 1a, 1b, 1c). Una dieta rica en vegetales con un alto contenido de compuestos fitoquímicos es recomendable para la prevención de algunas enfermedades y el cáncer (Preciado-Rangel et al. 2021), de ahí la importancia de incrementar la biosíntesis de estos compuestos antes de su cosecha y su posterior consumo. La mayor calidad nutracéutica y actividad antioxidante en las lechugas tratadas con Se, puede estar relacionado con una mayor actividad enzimática antioxidante del glutatión peroxi- 

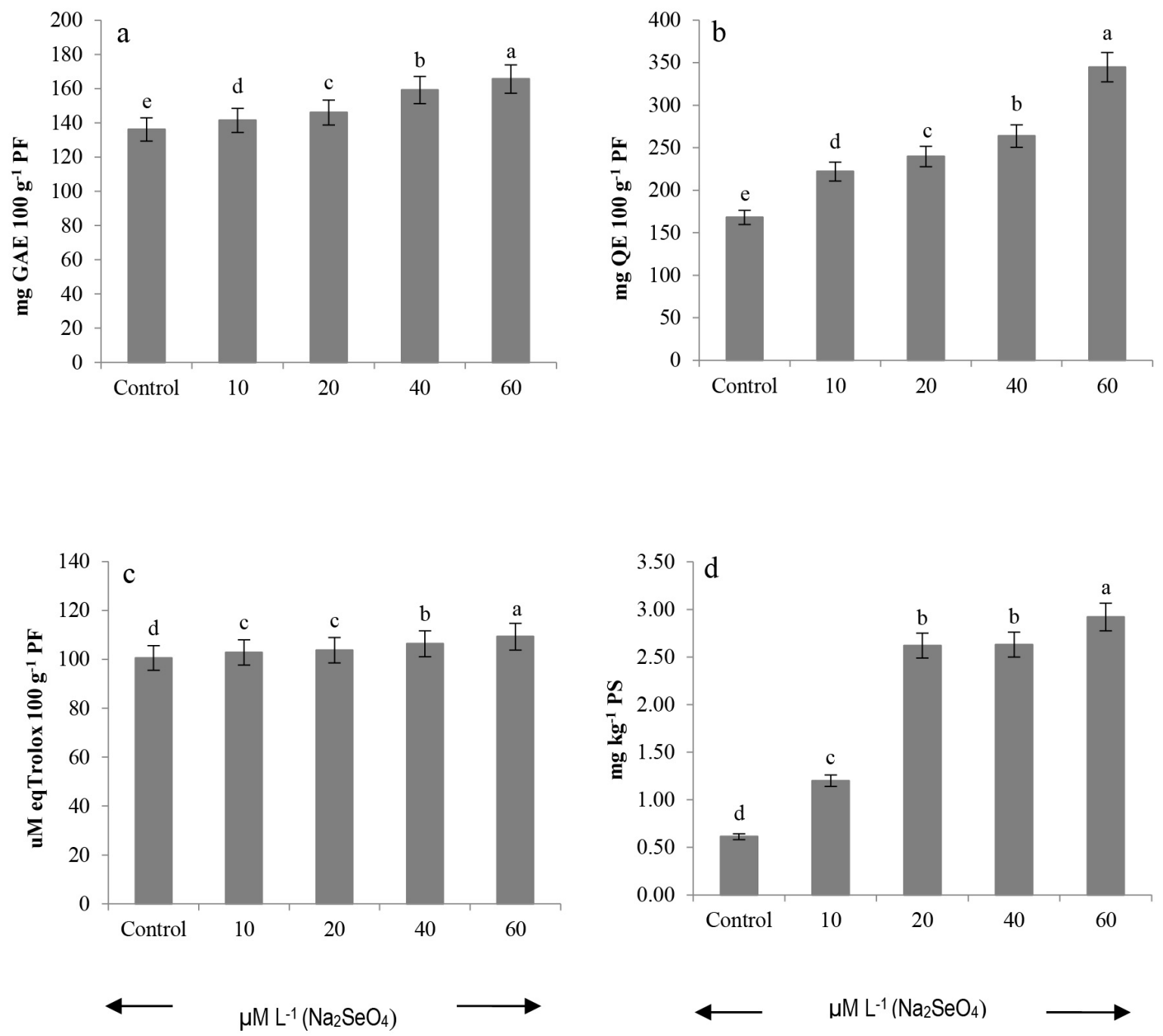

Figura 1. Efecto del Se sobre el contenido de compuestos fenólicos (a), flavonoides (b), capacidad antioxidante (c) y concentración de selenio (d) en plantas de lechuga. *Las columnas con letras diferentes difieren estadísticamente entre ellas (Tukey P $\leq 0.05$ ).

dasa ya que él Se forma parte de sus cuatro centros catalíticos (Hawrylak-Nowak et al. 2018). Además, se ha demostrado que este elemento regula la ruta biosintética de los fenilpropanoides, que conduce a una mayor biosíntesis de metabolitos secundarios, los cuales determinan las características organolépticas y la capacidad antioxidante (Mimmo et al. 2017). Esta respuesta también se ha reportado en investigaciones desarrolladas en diversos cultivos, con diferentes fuentes de Se y formas de aplicación (Zahedi et al. 2019, Groth et al. 2020).

\section{Contenido de Se en lechuga}

La bioacumulación de Se en las hojas de lechuga se incrementó de manera proporcional a la dosis asperjada (Figura 1d). La mayor acumulación de este elemento correspondió a la mayor dosis utilizada $\left(60 \mu \mathrm{M} \mathrm{L}^{-1}\right)$, superando un $79 \%$ al tratamiento testigo. Desde su descubrimiento en 1817, muchos estudios han confirmado la esencialidad del Se para la salud humana, y su rol en la prevención de enfermedades crónicas, cardiovasculares, y como componente esencial de más de 25 enzimas (El-Ramady et al. 2020). No obstante, el aporte de Se que se 
obtiene de los alimentos de origen vegetal, en algunas regiones del mundo, no es el suficiente para suplir la ingesta diaria que fluctúa entre $55-400 \mu \mathrm{g}$ día $^{-1}$ por adulto (Stefani et al. 2020). Debido a la baja eficiencia de la fertilización edáfica, la aspersión foliar con Se, es considerado como el método más efectivo para la biofortificación de los cultivos (Deng et al. 2019), incluido los sistemas hidropónicos los cuales son muy eficientes (Smoleń et al. 2014). Estudios previos muestran que la biofortificación aumenta significativamente la cantidad de elementos esenciales en la parte comestible de la planta (da Silva et al. 2020), la cual puede aumentar en los cultivos biofortificados con Se hasta $30 \%$ con respecto a los cultivos no tratados (Zhu et al. 2017). Nuestros resultados sugieren que el consumo de lechuga biofortificadas con $10 \mu \mathrm{M}$ de Se ( 100 g) aportan la cantidad promedio de Se de $120 \mu \mathrm{g} \mathrm{dia}^{-1}$, y el consumo de lechugas biofortificadas con $60 \mu \mathrm{M}$ de Se ( 100 g), aportan la cantidad media recomendada en la ingesta diaria $\left(\sim 250 \mu \mathrm{g} \mathrm{dia}^{-1}\right)$. Con lo anterior, la aspersión foliar con Se en lechuga, surge como una alternativa para mejorar la calidad nutracéutica de un cultivo de periodo corto, de rápido crecimiento, y con la capacidad de acumular Se en sus tejidos, dejándola como una opción más para disminuir la deficiencia de Se en humanos.

\section{LITERATURA CITADA}

Brand-Williams W, Cuvelier ME, Berset C (1995) Use of a free radical method to evaluate antioxidant activity. LWT-Food Science ad Technology 28: 25-30.

Calvo NIR, Echeverría HE, Rozas HS (2008) Comparación de métodos de determinación de nitrógeno y azufre en planta: Implicancia en el diagnóstico de azufre en trigo. Ciencia del Suelo 26: 161-167.

Chatepa LEC, Masamba KG (2020) Proximate and phytochemical composition of selected indigenous leafy vegetables consumed in Malawi. African Journal of Food Science 14: 265-273.

da Silva DF, Cipriano PE, de Souza RR, Siueia M, Faquin V, de Souza-Silva ML, Guilherme LRG (2020) Biofortification with selenium and implications in the absorption of macronutrients in Raphanus sativus $\mathrm{L}$. Journal of Food Composition and Analysis 86: 103382. DOI: 10.1016/j.jfca.2019.103382.

Deng XF, Zhao ZQ, Han ZY, Huang LQ, Lv CH, Zhang ZH, Liu XW (2019) Selenium uptake and fruit quality of pear (Pyrus communis L.) treated with foliar Se application. Journal of Plant Nutrition Soil Science 182: 637-646.

El-Ramady H, Faizy S ED, Abdalla N, Taha, H, Domokos-Szabolcsy É, Fari M, Brevik EC (2020) Selenium and Nano-Selenium Biofortification for Human Health: Opportunities and Challenges. Soil Systems 4: 57. DOI: $10.3390 /$ soilsystems 4030057 .

Emelike N, Ujong A, Achinewhu SI (2020) Proximate composition, mineral bioavailability and functional properties of defatted and undefatted avocado pear (Persia americana) seed flours. Asian Food Science Journal 17: $1-10$.

García-Márquez V, Morelos-Moreno Á, Benavides-Mendoza A, Medrano-Macías J (2020) lonic selenium and nanoselenium as biofortifiers and stimulators of plant metabolism. Agronomy 10: 1399. DOI: 10.3390/agro nomy10091399.

Garcia-Nava MA (2009) Cuantificación de fenoles y flavonoides totales en extractos naturales. Universidad Autónoma de Querétaro. Revista Academica 1: 1-4.

Gaucin-Delgado JM, Hernandez-Montiel LG, Sanchez-Chavez E, Ortega-Ortiz H, Fortis-Hernandez M, ReyesPéRez JJ, Preciado-Rangel P (2020) Agronomic biofortification with selenium improves the yield and nu- 
traceutical quality in tomato under soilless conditions. Notulae Botanicae Horti Agrobotanici Cluj-Napoc 48: 1221-1232.

Groth S, Budke C, Neugart S, Ackermann S, Kappenstein FS Daum D, Rohn S (2020) Influence of a Selenium Biofortification on Antioxidant Properties and Phenolic Compounds of Apples (Malus domestica). Antioxidants 9: 187. DOI: 10.3390/antiox9020187.

Gupta M, Gupta S (2017) An overview of selenium uptake, metabolism, and toxicity in plants. Frontiers in Plant Science 7: 2074. DOI: 10.3389/fpls.2016.02074.

Hawrylak-Nowak B, Dresler, S, Rubinowska K, Matraszek-Gawron R, Woch W, Hasanuzzaman M (2018) Selenium biofortification enhances the growth and alters the physiological response of lamb's lettuce grown under high temperature stress. Plant Physiology and Biochemistry 127: 446-456.

Helrich K (1990) Official Methods of Analysis of the Association of Official Analytical Chemists, 15th ed. AOAC. Arlington, VA, USA. 1059p.

Hossain A, Skalicky M, Brestic M, Maitra S, Sarkar S, Ahmad Z, Laing AM (2021) Selenium Biofortification: Roles, Mechanisms, Responses and Prospects. Molecules 26: 881. DOI: 10.3390/molecules26040881.

Khan N, Ahmed MJ, Shah SZA (2019) Comparative analysis of mineral content and proximate composition from chilli pepper (Capsicum annuum L.) germplasm. Pure Applied Biology 8: 1338-1347.

Kim M J, Moon Y, Tou JC, Mou B, Waterland NL (2016) Nutritional value, bioactive compounds and health benefits of lettuce (Lactuca sativa L.). Journal of Food Composition and Analysis 49: 19-34.

Kleine-Kalmer R, Profeta A, Daum D, Enneking U (2021) Willingness to purchase selenium-and iodine-biofortified apples-a discrete choice analysis with German consumers. Nutrients 13: 1625. DOI: 10.20944/preprints202 103.0371.v1.

Lapaz A dM, Santos LFdM, Yoshida C H P, Heinrichs R, Campos M, Reis ARd (2019) Physiological and toxic effects of selenium on seed germination of cowpea seedlings. Bragantia 78: 498-508.

Lee M, Rivard C, Pliakoni E, Wang W, Rajashekar C (2021) Supplemental UV-A and UV-B affect the nutritional quality of lettuce and tomato: Health-Promoting phytochemicals and essential nutrients. American Journal of Plant Sciences 12: 104-126.

Medina-Lozano I, Bertolín JR, Díaz A (2021) Nutritional value of commercial and traditional lettuce (Lactuca sativa L.) and wild relatives: Vitamin C and anthocyanin content. Food Chemistry 359: 129864. DOI: 10.1016/j.foodchem.2021.129864.

Mimmo T, Tiziani R, Valentinuzzi F, Lucini L, Nicoletto C, Sambo P, Cesco S (2017) Selenium Biofortification in Fragaria x ananassa: Implications on Strawberry Fruits Quality, Content of Bioactive Health Beneficial Compounds and Metabolomic Profile. Frontiers in Plant Science, 8: 1887. DOI: 10.3389/fpls.2017.01887.

Moussa MID, Alashi A M, Sossa-Vihotogbe CNA, Akponikpe PBI, Baco MN, Djènontin AJ, Akissoé NH (2020) Proximate composition, mineral profi le and trypsin-inhibitory activity of West African leafy vegetables: infl uence of urea micro-dosing and harvest time. Polish Journal of Food Nutrition Sciences 70: DOI: $10.31883 /$ pjfns/119674.

Oliveira VCd, Faquin V, Guimarães KC, Andrade FR, Pereira J, Guilherme LRG (2018) Agronomic biofortification of carrot with selenium. Ciência e Agrotecnologia 42: 138-147.

Puccinelli M, Malorgio F, Pezzarossa B (2017) Selenium Enrichment of Horticultural Crops. Molecules 22: 933. DOI: 10.3390/molecules22060933. 
Preciado-Rangel P, Hernández-Montiel LG, Valdez-Cepeda RD, de la Cruz-Lázaro E, Lara-Capistrán L, MoralesMorales B, Gaucin-Delgado JM (2021) Biofortification with selenium increases bioactive compounds and antioxidant capacity in tomato fruits. Terra Latinoamericana 39: 1-10. DOI: 10.28940/terra.v39i0.979.

Rahim F P, Rocio CG, Adalberto BM, Lidia Rosaura SC, Maginot NH (2020) Agronomic Biofortification with Selenium in Tomato Crops (Solanum lycopersicon L. Mill). Agriculture 10: 486. DOI: 10.3390/agriculture10100486.

Rayman MP (2020) Selenium intake, status, and health: a complex relationship. Hormones 19: 9-14.

Ryant P, Antošovský J, Adam V, Ducsay L, Škarpa P, Sapáková E (2020) The importance of selenium in fruit nutrition. In: Srivastava AK, Hu C (eds.) Fruit Crops. Elsevier. England. pp: 241-254.

Sabatino L, Ntatsi G, lapichino G, D’Anna F, De Pasquale C (2019) Effect of Selenium Enrichment and Type of Application on Yield, Functional Quality and Mineral Composition of Curly Endive Grown in a Hydroponic System. Agronomy 9: 207. DOI: 10.3390/agronomy9040207.

Schiavon M, Nardi S, dalla-Vecchia F, Ertani A (2020) Selenium biofortification in the 21st century: status and challenges for healthy human nutrition. Plant and Soil 453: 245-270.

Smoleń S, Kowalska I, Sady W (2014) Assessment of biofortification with iodine and selenium of lettuce cultivated in the NFT hydroponic system. Scientia Horticulturae 166: 9-16.

Stefani S, Halim L, Andayani DE, Witjaksono F (2020) Selenium in Hyperthyroidism. World Nutrition Journal 3: 24-37.

Steiner AA (1961) A universal method for preparing nutrient solutions of a certain desired composition. Plant and Soil 15: 134-154.

Wang M, Ali F, Wang M, Dinh QT, Zhou F, Bañuelos GS, Liang D (2020) Understanding boosting selenium accumulation in Wheat (Triticum aestivum L.) following foliar selenium application at different stages, forms, and doses. Environmental Science Pollution Research 27: 717-728.

White PJ (2016) Selenium accumulation by plants. Annals of Botany 117: 217-235.

Willers J, Heinemann M, Bitterlich N, Hahn A (2015) Intake of minerals from food supplements in a German population-a nationwide survey. Food Nutrition Sciences 6: 205-215.

Wu Z, Bañuelos GS, Lin ZQ, Liu Y, Yuan L, Yin X, Li M (2015) Biofortification and phytoremediation of selenium in China. Frontiers in Plant Science 6: DOI: 10.3389/fpls.2015.00136.

Zahedi S M, Hosseini M S, Daneshvar Hakimi Meybodi N, Teixeira da Silva JA (2019) Foliar application of selenium and nano-selenium affects pomegranate (Punica granatum cv. Malase Saveh) fruit yield and quality. South African Journal of Botany 124: 350-358.

Zapata-Vahos IC, Rojas-Rodas F, David D, Gutierrez-Monsalve JA, Castro-Restrepo D (2020) Comparison of antioxidant contents of green and red leaf lettuce cultivated in hydroponic systems in greenhouses and conventional soil cultivation. Revista Facultad Nacional de Agronomía Medellín 73: 9077-9088.

Zhu Z, Chen Y, Shi G, Zhang X (2017) Selenium delays tomato fruit ripening by inhibiting ethylene biosynthesis and enhancing the antioxidant defense system. Food Chemistry 219: 179-184. 